\title{
DESARROLLO E IMPLEMENTACIÓN DE TECNOLOGÍAS BIOMÉDICAS PARA LA TELEREHABILITACIÓN FUNCIONAL
}

\section{DEVELOPMENT AND IMPLEMENTATION OF BIOMEDICAL TECHNOLOGIES FOR FUNCTIONAL TELEREHABILITATION}

\author{
PhD. Javier E. Sierra* , MsC. Boris Medina*, \\ MsC. Liliana Rodríguez ${ }^{* *}$, MsC. Claudia Pachón **, MsC. Meryene Barrios ** \\ * Universidad de Sucre, Facultad de Ingeniería, Grupo de Investigación e Innovación en \\ Electrónica (GINELECT). \\ Cra 28 \# 5-267, Sincelejo, Sucre, Colombia. \\ E-mail: \{javier.sierra, boris.medina $\} @$ unisucre.edu.co. \\ *** Corporación Universitaria Antonio José de Sucre, Facultad de Ciencias de la Salud, \\ Programa de Fisioterapia, Grupo de Investigación Fisioterapia en Movimiento (FIMOV). \\ Carrera 21 \# 25-59, Sincelejo, Sucre, Colombia. \\ E-mail: \{liliana_rodiguez, claudia_pachon, meryene_barrios\}@ corposucre.edu.co.
}

Resumen: El presente artículo muestra el desarrollo e implementación de un sistema de telerehabilitación funcional empleando entornos virtuales interactivos y tecnologías biomédicas en pacientes con secuelas de parálisis cerebral con limitación en los movimientos de las extremidades superiores e inferiores. El sistema facilita la intervención terapéutica empleando la telerehabilitación a través de videojuegos con interfaz de adquisición de movimientos. La plataforma se desarrolló en un lenguaje de programación de fácil configuración, de tal forma que los terapeutas puedan cambiar y ajustar los retos de cada intervención terapéutica de forma interactiva, amigable y divertida, acorde al progreso y motivando al paciente a continuar con su evolución. Los resultados muestran mejora en niños con parálisis cerebral, con limitaciones en movimiento a nivel de miembros superiores o inferiores.

Palabras clave: Telerehabilitación, discapacidad, realidad virtual, videojuegos, biomédica.

\begin{abstract}
We show the development and implementation of a functional telerehabilitation system using interactive virtual environments and biomedical technologies in patients with sequelae of cerebral palsy with limited movement of the upper and lower extremities. The system facilitates therapeutic intervention using telerehabilitation through video games with movement acquisition interface. The platform was developed in an easy-to-configure programming language, so that therapists can change and adjust the challenges of each therapeutic intervention in an interactive, friendly and fun way, according to progress and motivating the patient to continue with their evolution. The results show improvement in children with cerebral palsy, with limitations in movement at the level of upper and lower limbs.
\end{abstract}

Keywords: Telerehabilitation, disability, virtual reality, videogames, biomedical. 


\section{INTRODUCCIÓN}

La telemedicina permite aprovechar las tecnologías de la información, la imagen de video y las telecomunicaciones para ofrecer mejores servicios de salud. Esto ha permitido la evolución de los equipos biomédicos. La telerehabilitación es considerada un subcomponente de la telemedicina y se refiere a la aplicación clínica de servicios consultivos, preventivos, diagnósticos y terapéuticos a través de tecnologías interactivas bidireccionales (Barrios, Rodriguez, Pachón, Medina, \& Sierra, 2019).

La incorporación de procedimientos de intervención con videojuegos ha demostrado ser más eficaz y motivadora para mejorar las deficiencias motoras en los pacientes, obteniendo beneficios en su calidad de vida (Borja, 2017). Los últimos años han sido testigos de un aumento en telerehabilitación y sistemas de salud remotos que emplean dispositivos y tecnologías emergentes reutilizables de bajo costo para vigilar los aspectos biológicos y biocinemáticos de los seres humanos (Li et al., 2015). Estos dispositivos inteligentes acompañados con el procesamiento de gran contenido de información que pueden suministrar estas tecnologías, son hoy en día parte viva de las arquitecturas asistidas en múltiples ámbitos y pueden ser adaptados y personalizados dentro de sistemas inteligentes para la medición, monitoreo y evaluación en la rehabilitación de pacientes.

El presente trabajo muestra el sistema de telerehabilitación SmartRehab, el cual permite la aplicación de protocolos de rehabilitación especialmente diseñados para pacientes con parálisis cerebral, teniendo en cuenta las características de los pacientes y una programación sencilla que permite la configuración acorde al progreso de los pacientes.

\subsection{Telerehabilitación}

La telerehabilitación es una alternativa a los servicios de rehabilitación ambulatorios de atención habitual (Dorsey, George, Leff, \& Willis, 2013). La telerehabilitación se ha ampliado drásticamente en los últimos años como resultado de los avances en la tecnología, el aumento de la velocidad de las telecomunicaciones y la disminución de los costos de hardware y software informático (De Araujo et al., 2019).

La telerehabilitación puede dividirse en tres categorías principales: telerehabilitación basada en imágenes, telerehabilitación basada en sensores y telerehabilitación basada en tecnologías virtuales (Langberg H, Lindahl MP, Kidholm K, 2014). Las terapias a través de un escenario virtual parecen fomentar el aprendizaje motor, la retención de las habilidades aprendidas, y la transferencia de habilidades a situaciones del mundo real (Massetti et al., 2014; Pereira, Barbosa, \& Goncalves, 2019). $\mathrm{La}$ realidad virtual (RV) se emplea en la rehabilitación física con el objeto de mejorar la función motora. Actualmente, esta tecnología se aplica con más frecuencia en patologías de origen neurológico (ictus, enfermedad de Parkinson, lesiones medulares, parálisis cerebral infantil, entre otras), logrando resultados de mejoría en la intervención y motivación de los pacientes para conseguir un elevado nivel de mejora funcional (Barrios et al., 2019; Laver, George, Thomas, Deutsch, \& Crotty, 2015).

El entorno VR depende de la tecnología (hardware y software) utilizada y teniendo en cuenta el grado de inmersión del paciente en el entorno, estos sistemas pueden ser inmersivos y no inmersivos. En los sistemas inmersivos el usuario está integrado totalmente dentro del ambiente virtual, viendo solo las imágenes generadas por el ordenador, bloqueándose el resto del mundo físico; dentro de estos sistemas se encuentran Glasstrom, IREX, Playstation EyeMotion. En los sistemas no inmersivos o semi-inmersivos el usuario percibe parte del mundo real y parte del mundo-entorno virtual; no hay una inmersión total en el entorno virtual. En la actualidad, los sistemas no inmersivos más utilizados son Virtual Teacher, Xbox, Cyberglobe, Virtual Reality Motion, Pneumoglobe y Nintendo-Wii (Viñas-Diz \& Sobrido-Prieto, 2016).

\section{ESTADO DEL ARTE}

Según (Ramírez et al., 2014), la terapia basada en realidad virtual cuenta con un contexto funcional, concreto y estimulante para los pacientes, trayendo un beneficio directo tanto al paciente como al terapeuta por la adaptabilidad que tienen estos sistemas. Los autores realizan pruebas en pacientes entre 6 y 12 años de edad con discapacidades en extremidades superiores para su rehabilitación, basada en una arquitectura de hardware y software mediante videojuegos que inducen actividades físicas particulares en el paciente, procurando su atención mediante contenido dinámico y entretenido de forma interactiva. Así mismo, (Yagüe Sebastián, Yagüe Sebastián, Lekuona Amiano, \& Sanz Rubio, 2016) también confirman 
lo beneficioso e interesante de la aplicación del videojuego mediado por las nuevas tecnologías, para el tratamiento fisioterapéutico de la parálisis cerebral.

Por su parte, (Bayón \& Martínez, 2010) en su artículo sobre rehabilitación del ictus mediante realidad virtual hizo una revisión donde describe los principales sistemas inmersivos y no inmersivos de realidad virtual utilizados en el tratamiento de pacientes con ictus, indagando además sobre los mecanismos de neuroplasticidad y reorganización cortical.

(Farreny et al., 2012) realizaron una investigación titulada Play for health $(\mathrm{P} 4 \mathrm{H})$ : una nueva herramienta en telerehabilitación. El P4H es un sistema de telerehabilitación abierto y de bajo costo basado en el uso de videojuegos y métodos de interacción para mejorar déficits físicos $\mathrm{y}$ cognitivos. El objetivo de la investigación fue presentar $\mathrm{P} 4 \mathrm{H}$, valorar su aceptación y describir la repercusión organizativa que ha supuesto su introducción en el servicio de rehabilitación.

En los últimos años se ha visto como el nacimiento de nuevas tecnologías emergente de realidad virtual son usadas como herramientas de telerehabilitación para mejorar las alternativas y opciones para nuevos tratamientos. De este modo es posible encontrar propuestas como:

(Siyli, Akarun, \& Arica, 2013) en su investigación propone un sistema que permite a las personas con discapacidades, realizar ejercicios en la comodidad de sus hogares e independientemente de las restricciones. Emplean el Kinect para adquirir los modelos corporales de los pacientes, y a través de procesamiento de imágenes analizan las diferentes situaciones. Una investigación realizada por (Casanova, Mario; Muñoz, John;Henao, 2015), señala un sistema para la rehabilitación física de pacientes con múltiples patologías, a través de dinámicas con videojuegos, y se analizan los movimientos de los pacientes usando un software específico. En este estudio se basaron en el uso del sensor Kinect, diseñado para divertir al paciente en su terapia a través de exergames y al mismo tiempo le proporciona al especialista un registro y análisis de datos de captura de movimiento (MoCap).

(Couto Soares, Vieira, \& Gabriel, 2013) en su investigación proponen un programa de rehabilitación en el hogar basado en videojuegos, empleando el sensor Kinect, de Microsoft. Los tratamientos de rehabilitación están dirigidos a las enfermedades cardiovasculares (ECV), los cuales contribuyen en gran medida a la mortalidad y la morbilidad a nivel mundial. La propuesta incluye programas basados en el ejercicio, adaptados a cada paciente, que tienen como objetivo restaurar y mejorar la función cardíaca, disminuir la discapacidad, reducir la mortalidad, mejorar la calidad de vida y reducir los costos del tratamiento.

(Pachoulakis, Xilourgos, Papadopoulos, \& Analyti, 2017) implementan una plataforma de fisioterapia basada en Kinect adaptada a los pacientes con enfermedad de Parkinson (PD); el sensor Kinect se emplea para extraer datos esqueléticos en $3 \mathrm{D}$ en tiempo real de un paciente en ejercicio.

\section{MATERIALES Y MÉTODOS}

\subsection{Sensor Kinect}

Kinect es un sensor creado y lanzado por Microsoft en 2010, construido para revolucionar la forma en que las personas juegan y experimentan el entretenimiento (Murillo, 2018). Con Kinect, las personas son capaces de interactuar con el juego mediante movimientos de su propio cuerpo. Este sensor incorpora varios dispositivos de detección avanzados. En particular, contiene un sensor de profundidad, una cámara RGB y una matriz de cuatro micrófonos que proporcionan capacidades de reconocimiento facial y de movimiento en 3D del cuerpo completo. La clave de esta tecnología radica en la comprensión del lenguaje corporal humano; la interfaz debe entender primeramente el movimiento antes de que pueda responder. Esto ha sido un campo activo de investigación en visión por computador, ya que permite que el ordenador detecte directamente la señal en 3D (profundidad) del usuario, el audio y el entorno, facilitando mucho la tarea de reconocimiento, que traducido a un formato, los desarrolladores pueden utilizarlo para construir nuevas experiencias.

\subsection{Plataforma de desarrollo}

Scratch ${ }$ es un lenguaje de programación visual desarrollado por el Grupo Lifelong Kindergarten del MIT Media Lab (Scratch, 2018). Su principal característica consiste en que permite el desarrollo de habilidades mentales mediante el aprendizaje de la programación sin tener conocimientos profundos sobre el código. Sus características ligadas al fácil entendimiento del pensamiento computacional han hecho que sea muy difundido en la educación de niños, adolescentes y adultos. 
Scratch permite a los usuarios usar programación dirigida por eventos con múltiples objetos activos llamado sprites. Los sprites pueden pintarse como gráficos vectoriales o mapa de bits, desde la propia web de Scratch usando un simple editor que es parte del proyecto, o pueden también importarse desde fuentes externas incluyendo "webcamera". El código fuente de Scratch 1.x es bajo licencias GPLv2 y "Scratch Source Code License".

\subsection{Sistema SmartRehab}

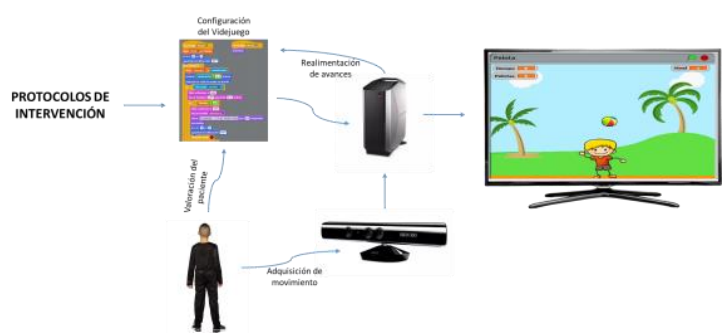

Fig. 1. Sistema SmartRehab

El sistema de telerehabilitación en entorno de realidad virtual desarrollado "SmartRehab" es mostrado en la Figura 1, en donde se observan los elementos principales. El sistema parte del diseño de protocolos de intervención que tienen en cuenta las características del paciente, luego de haberle realizado una valoración. Con la valoración se establece el protocolo de intervención y de esta forma es posible realizar ajustes a los retos que tendría que afrontar el paciente en el videojuego. Los movimientos del paciente son recibidos a través del Kinect, el cual envía los datos al software desarrollado empleando Scratch. El software a través de codificación interna, que conecta con SDK del Kinect, realiza el procesamiento de la imagen para la toma de decisiones. El código de uno de los videojuegos es mostrado en la Figura 2 (Canasta), permite fácil configuración, de tal forma que los terapeutas puedan cambiar y ajustar los retos de cada intervención terapéutica de forma interactiva, amigable y divertida, acorde al progreso y motivando al paciente a continuar con su evolución.

La plataforma dispone de una colección de 4 videojuegos (Rana, Canasta, Básquet, Voleibol) teniendo en cuenta protocolos de rehabilitación diseñados especialmente para pacientes con parálisis cerebral. Cada ejercicio emplea un patrón de movimiento con demandas de procesamiento muy ligeras para cálculos en tiempo real. Los resultados son guardados para ser empleado en el mejoramiento del protocolo de rehabilitación y de los videojuegos.

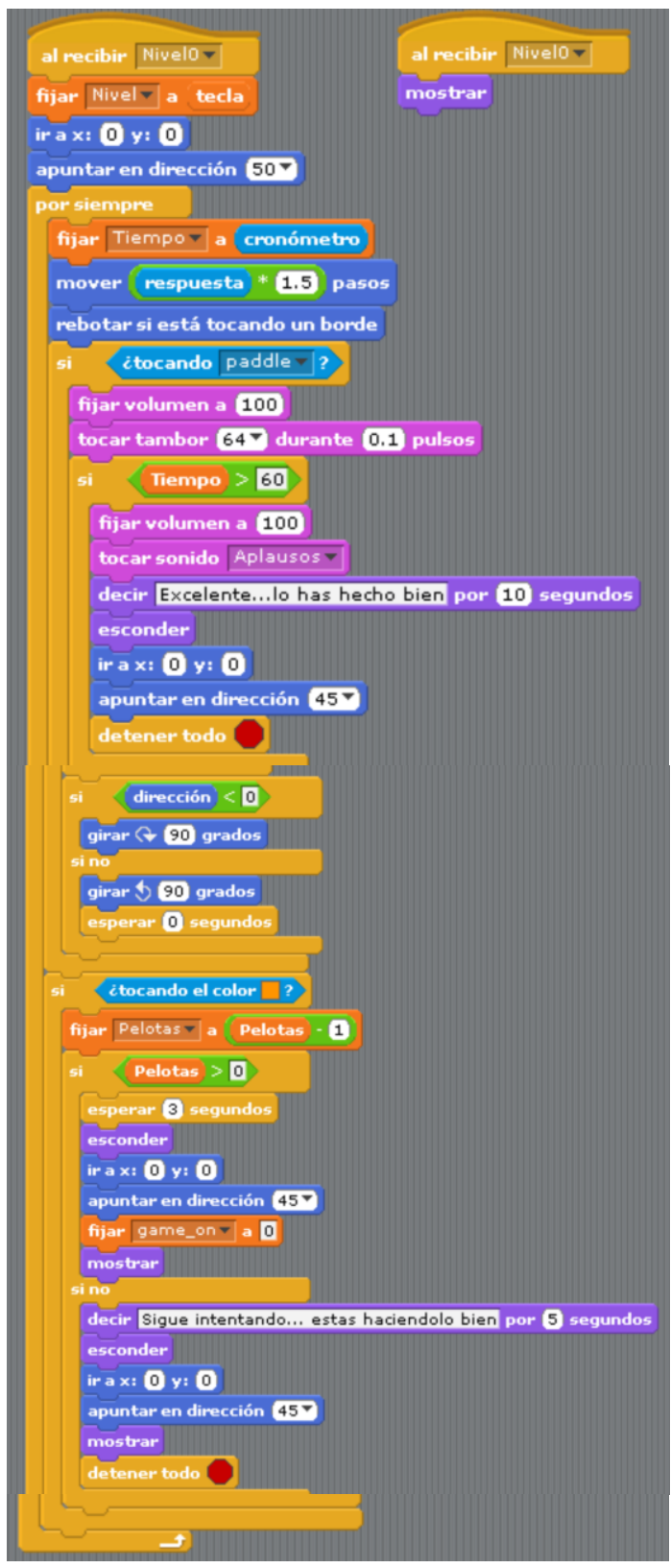

Fig. 2. Código Videojuego Canasta

\section{RESULTADOS}

Para la validación del sistema desarrollado, se realizaron pruebas con niños con parálisis cerebral. La investigación se realizó en las instalaciones del laboratorio de neurorehabilitación de la Corporación Universitaria Antonio José de Sucre, en los niños con limitaciones del movimiento de las extremidades superiores por secuela de parálisis cerebral, cuyos padres aceptaron voluntariamente a 
través de consentimiento informado que sus hijos participen en el estudio. La muestra es de tipo no probabilístico, y dependió del número de niños que asistieran a las instalaciones y que cumplan con los siguientes criterios:

Criterios de inclusión:

- $\quad$ Edad comprendida entre 5 y 12 años.

- Niños cognitivamente altos

- Estrato socioeconómico 1 y 2

- Gross motor de nivel 1 y 2 .

Criterios de exclusión:

- Pacientes con lesiones auditivas y visuales.

- Pacientes con ataxias.

- Pacientes con lesiones traumáticas no resueltas.

Inicialmente, se evaluó la condición de discapacidad en la que se encontraban los niños con secuelas de parálisis cerebral con alteraciones de extremidades superiores, para determinar el nivel de discapacidad en la que se encuentran y así poder concretar desde allí su plan de intervención a través del videojuego. Se aplicó el test Gross Motor para valorar la condición del niño.

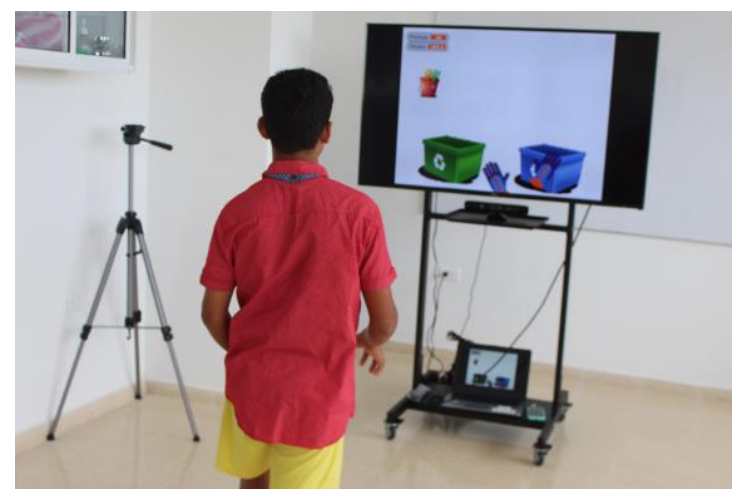

Fig. 3. Paciente 1 con parálisis cerebral empleando SmartRehab

Se desarrollaron 20 sesiones de trabajo con cada paciente, efectuando pruebas pilotos con la plataforma biomédica virtual e interactiva del protocolo de las extremidades superiores dirigido a los pacientes que cumplan con los criterios de inclusión. Las pruebas se efectuaron bajo la supervisión de profesionales capacitados para ello, fisioterapeutas previamente entrenados en los videojuegos para la satisfacción total de las intervenciones realizadas.

Una vez finalizada las sesiones, se volvió a evaluar la condición del niño para mirar el nivel de evolución en el control postural a través de los videojuegos en los niños con secuelas de parálisis cerebral en las extremidades superiores. La Figura 3 y la Figura 4 muestran el uso de la plataforma, conformada por un computador, un televisor, un Kinect y el sistema de realidad virtual.

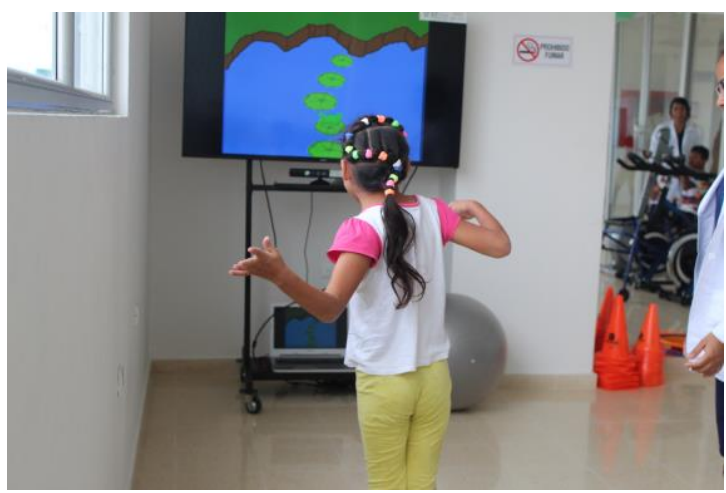

Fig. 4. Paciente 2 con parálisis cerebral empleando SmartRehab

Se trabajó con 3 niños con diagnóstico de parálisis cerebral, entre edades de 10 y 15 años, cumpliendo con los criterios de la inclusión, gross motor I y II, de los cuales dos eran de sexo masculino y una de sexo femenino.

En la Figura 5 se muestra los resultados de la valoración inicial, en donde se observa la puntuación en el paciente \# 1 en decúbito y rolado (dimensión A) el niño alcanza un 90\%; en sedente (dimensión B) 91\%; en gateo y de rodillas (dimensión C) llegó a una puntuación del 64\%; en bipedestación (estar de pie) (dimensión D) arrojó un puntaje de $66 \%$, al igual que en marcha, carrera y salto (dimensión E) con un porcentaje de $36 \%$. En el paciente \# 2 en decúbito y rolado el niño alcanza un $80 \%$; en sedente $93 \%$; en gateo y de rodillas llego a una puntuación del $88 \%$; en bipedestación arrojó un puntaje de $79 \%$, al igual que en marcha, carrera y salto con un porcentaje de $86 \%$. En el paciente \# 3 en decúbito y rolado el niño alcanza una puntuación de $96 \%$; en sedente el niño tiene una puntuación de $98 \%$; en gateo y de rodillas equivale a un $80 \%$; en bipedestación arrojó un puntaje que corresponde al $64 \%$, al igual que en marcha, carrera y salto un porcentaje de $29 \%$. Lo que indica que los pacientes \#1 y \#2 presentan grandes dificultades para las dimensiones de bipedestación; y marcha, carrera y salto. 


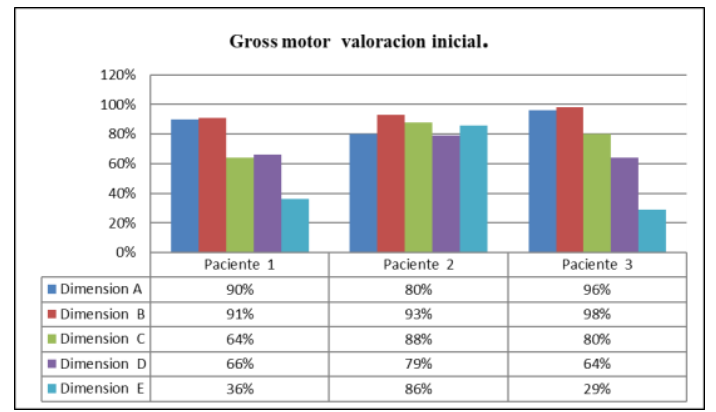

Fig. 5. Valoración inicial, gross motor

La Figura 6 muestra el resultado de la valoración final en la gross motor. En el paciente \# 1 en decúbito y rolado el niño alcanza un porcentaje de $100 \%$; en sedente un $96 \%$; en gateo y de rodillas llego un 66\%; en bipedestación arrojó un porcentaje de $76 \%$, y en marcha, carrera y salto $55 \%$. En el paciente \# 2 en decúbito y rolado el niño alcanza un porcentaje de $92 \%$; en sedente un $95 \%$; en gateo y de rodillas llego un $90 \%$; en bipedestación arrojó un porcentaje de $100 \%$, y en marcha, carrera y salto $100 \%$. En el paciente \# 3 en decúbito y rolado el niño alcanza un porcentaje de 96\%; en sedente el niño tiene un 100\%; en gateo y de rodillas llego a un 92\%; en bipedestación arrojó un $74 \%$, al igual que en marcha, carrera y salto con un porcentaje de $40 \%$. Después de la prueba piloto los pacientes mejoraron en todas las dimensiones de la gross motor, en especial en la bipedestación

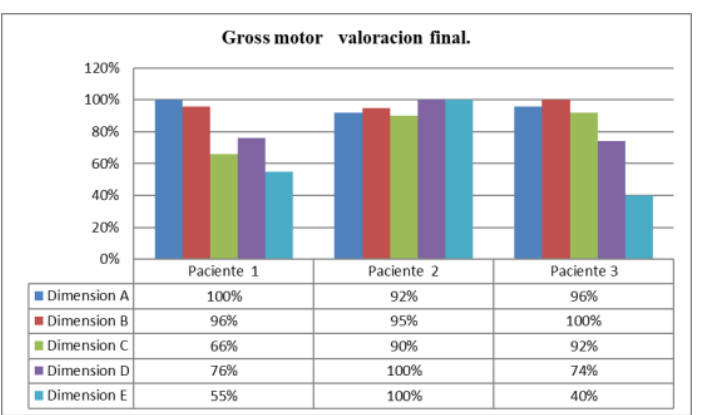

Fig. 6. Valoración final, gross motor

Se puede apreciar al realizar comparación antes y después de las pruebas, que el protocolo de intervención basado en videojuegos mejora las limitaciones en las extremidades superiores y en el control postural. Todos los pacientes mostraron evolución en cada escala e ítem de valoración, lo que indica que hubo resultados satisfactorios y por ende la estrategia de la combinación de intervención fue benéfica.

\section{CONCLUSIONES}

El sistema SmartRehab desarrollado facilita la intervención terapéutica empleando la telerehabilitación a través de videojuegos, ya que posee una interfaz que permite que los terapeutas puedan cambiar y ajustar los retos de cada intervención de forma interactiva, amigable y divertida, acorde al progreso y motivando al paciente a continuar con su evolución. Mediante la experiencia realizada durante la intervención se pudo concluir que la modalidad de rehabilitación virtual a través de videojuegos con sensor Kinect es una alternativa de intervención a los pacientes con secuelas de parálisis cerebral pero aplicada con las diferentes técnicas de neurorehabilitación, ya que son buen complemento para la rehabilitación del paciente.

\section{RECONOCIMIENTO}

Los autores agradecen a la Corporación Universitaria Antonio José de Sucre CORPOSUCRE por la financiación del Proyecto SMARTREHAB en sus fases 1 y 2.

\section{REFERENCIAS}

Barrios, M., Rodriguez, L., Pachón, C., Medina, B., \& Sierra, J. E. (2019). Functional telerehabilitation based on interactive virtual environments as a rehabilitation proposal for patients with disabilities. Espacios, 40(25), 1-14. Retrieved from https://www.revistaespacios.com/a19v40n25/ a19v40n25p01.pdf

Bayón, M., \& Martínez, J. (2010). Rehabilitación del ictus mediante realidad virtual. Rehabilitación, 44(3), 256-260. https://doi.org/10.1016/j.rh.2009.11.005

Borja, B. (2017). Personas con discapacidad intelectual: Implementación de un programa de intervención para mejorar la calidad de vida a través de Xbox-Kinect. Universidad de Extremadura. Retrieved from http://dehesa.unex.es/bitstream/handle/10662 /6420/TDUEX_2017_Carbonell_Blanco.pdf? sequence $=1 \&$ isAllowed $=\mathrm{y}$

Casanova, Mario; Muñoz, John;Henao, O. L. D. (2015). Exergames como herramienta para la evaluación del equilibrio postural en un paciente con esclerosis múltiple. IEEE. Retrieved from https://www.researchgate.net/profile/John_M unoz4/publication/283546834_Exergames_as 
_a_tool_for_the_assessment_of_postural_bal ance_in_a_patient_with_multiple_sclerosis_ The_role_of_biomechanical_analysis_in_the _quantification_of_movement/links/57964ba $708 \mathrm{aeb}$

Couto Soares, J., Vieira, A., \& Gabriel, J. (2013). Assisted living: Home physiotherapy demo. In 2013 2nd Experiment@ International Conference (exp.at'13) (pp. 162-163). IEEE. https://doi.org/10.1109/ExpAt.2013.6703054

De Araujo, F. M. A., Viana Filho, P. R. F., Adad Filho, J. A., Fonseca Ferreira, N. M., Valente, A., \& Soares, S. F. S. P. (2019). A new approach of developing games for motor rehabilitation using Microsoft Kinect. In 2019 IEEE 7th International Conference on Serious Games and Applications for Health, SeGAH 2019. Institute of Electrical and Electronics Engineers Inc. https://doi.org/10.1109/SeGAH.2019.888245 7

Dorsey, E. R., George, B. P., Leff, B., \& Willis, A. W. (2013). The coming crisis: obtaining care for the growing burden of neurodegenerative conditions. Neurology, 80(21), 1989-1996. https://doi.org/10.1212/WNL.0b013e318293 e2ce

Farreny, M. A., Buen, M. C., Aguirrezabal, A., Ferriol, P., Tous, F., \& Alcalde, M. A. (2012). Play for health (P4H): una nueva herramienta en telerehabilitación. Rehabilitación, 46(2), 135-140. https://doi.org/10.1016/j.rh.2012.01.001

Langberg H, Lindahl MP, Kidholm K, D. B. (2014). Telerehabilitering. Videnskab. Retrieved from https://telemed.custompublish.com/getfile.ph p/3205551.357.sxwqucbfyc/Telerehabiliterin g+2014.pdf

Laver, K. E., George, S., Thomas, S., Deutsch, J. E., \& Crotty, M. (2015). Virtual reality for stroke rehabilitation. In K. E. Laver (Ed.), Cochrane Database of Systematic Reviews. Chichester, UK: John Wiley \& Sons, Ltd. https://doi.org/10.1002/14651858.CD008349 .pub3

Li, S., Pham, H. T., Karunarathne, M. S., Lee, Y. S., Ekanayake, S. W., \& Pathirana, P. N. (2015). A Mobile Cloud Computing Framework Integrating Multilevel Encoding for Performance Monitoring in Telerehabilitation. Mathematical Problems in Engineering, 2015. https://doi.org/10.1155/2015/617840

Massetti, T., Silva, T. D. da, Ribeiro, D. C., Malheiros, S. R. P., Ré, A. H. N., Favero, F.
M., ... Monteiro, C. B. de M. (2014). Motor learning through virtual reality in cerebral palsy - a literature review. Medical Express, 1(6), 302-306.

https://doi.org/10.5935/MedicalExpress.2014 .06 .04

Murillo, A. (2018). ¿Qué es el dispositivo Kinect?| Kinect for Developers. Retrieved November 27, 2019, from http://www.kinectfordevelopers.com/es/2012 /11/06/que-es-el-dispositivo-kinect/

Pachoulakis, I., Xilourgos, N., Papadopoulos, N., \& Analyti, A. (2017). Enrichment of a Kinect-based Physiotherapy and Assessment Platform for Parkinson's disease Patients. Advances in Image and Video Processing, 5(1), 31. https://doi.org/10.14738/aivp.51.2750

Pereira, L. B., Barbosa, D. D., \& Goncalves, R. S. (2019). Development of games controlled by kinect to spine physical therapy. In IEEE International Conference on Rehabilitation Robotics (Vol. 2019-June, pp. 193-197). IEEE Computer Society. https://doi.org/10.1109/ICORR.2019.877949 4

Ramírez, E., Moreno, F., Ojeda, J., Mena, C., Rodríguez, O., Rangel, J., \& Álvarez, S. (2014). Un Framework para la Rehabilitación Física en Miembros Superiores con Realidad Virtual. Revista Venezolana de Computación, 1(1), 8-16. Retrieved from http://saber.ucv.ve/ojs/index.php/rev_vcomp/ article/view/6931/6671

Scratch, F. (2018). Scratch - Imagine, Program, Share. Retrieved November 27, 2019, from https://scratch.mit.edu/

Siyli, R. D., Akarun, L., \& Arica, N. (2013). Physiotherapy guidance by motion analysis based on Hidden Markov Model. In 2013 21st Signal Processing and Communications Applications Conference (SIU) (pp. 1-4). IEEE. https://doi.org/10.1109/SIU.2013.6531499

Viñas-Diz, S., \& Sobrido-Prieto, M. (2016). Virtual reality for therapeutic purposes in stroke: A systematic review. Neurología (English Edition), 31(4), 255-277. https://doi.org/10.1016/j.nrleng.2015.06.007

Yagüe Sebastián, M. P., Yagüe Sebastián, M. M., Lekuona Amiano, A., \& Sanz Rubio, M. C. (2016). Los videojuegos en el tratamiento fisioterápico de la parálisis cerebral. Fisioterapia, 38(6), 295-302. https://doi.org/10.1016/j.ft.2015.11.005 